\title{
Aspectos Psicológicos da Fibromialgia - Revisão Integrativa
}

\author{
Érico Augusto Barreto Monteiro* \\ Luciene de Oliveira* \\ Walter Lisboa Oliveira**
}

\begin{abstract}
Resumo
O objetivo do trabalho foi apresentar um panorama descritivo de estudos relacionando aspectos psicológicos à fibromialgia. Foi realizada uma revisão integrativa a partir de levantamento bibliográfico nos bancos Scielo e Pepsic, considerando o período de 2015 a 2019. Foram encontrados 96 artigos e 19 foram selecionados segundo os critérios de inclusão. Os dados foram organizados, tabulados e submetidos a Análise Bibliométrica e de Conteúdo. Resultaram 13 artigos de pesquisa de campo, quatro de revisão bibliográfica e dois psicométricos. Seus conteúdos foram analisados em quatro categorias: "coping e estresse", "ansiedade", "depressão" e "outros aspectos psicológicos". Destacam-se dificuldades de adaptação à enfermidade, com consequências negativas como isolamento social, redução de atividades físicas, fadiga, interferência na vida sexual e no sono, além de estresse como consequência e agravante dos sintomas. Houve prevalência de estudos sobre ansiedade e depressão, com correlação positiva de ambas com a intensidade da dor e outros sintomas da fibromialgia.
\end{abstract}

Palavras-chave: Fibromialgia; Dor Crônica; Psicologia da Saúde.

\section{Psychological Aspects of Fibromyalgia - Integrative Review}

\begin{abstract}
.
The objective was to present a descriptive panorama of studies relating psychological aspects to fibromyalgia. An integrative review was conducted from a bibliographic survey in Scielo and Pepsic, considering the period from 2015 to 2019. Ninety-six articles were found and 19 were selected according to the inclusion criteria. Data were organized, tabulated, and submitted to Bibliometric and Content Analysis. The result were 13 field research articles, four bibliographic reviews and two psychometrics studies, and their contents were analyzed in four categories: "coping and stress", "anxiety", "depression" and "other psychological aspects". They highlight difficulties in adapting to the disease, with negative consequences such as social isolation, reduction of physical activities, fatigue, interference in sexual life and sleep, besides stress as a consequence and an aggravating of the symptoms. There was a prevalence of studies about anxiety and depression, with positive correlation of both with pain intensity and other symptoms of fibromyalgia.
\end{abstract}

Keywords: Fibromyalgia; Chronic Pain; Health Psychology.

* Graduanda (o) de Psicologia da Universidade Federal de Sergipe

** Prof. Adjunto da Universidade Federal de Sergipe e Tutor de Psicologia do Programa de Residência Multiprofissional Saúde do Adulto e do Idoso do $\mathrm{HU} / \mathrm{UFS}$. 


\section{Introdução}

A Fibromialgia (FM) é uma doença crônica caracterizada pela presença constante de dor, porém de causa ainda desconhecida. Pode ocorrer nos músculos, articulações ou em diversos pontos, gerando um estado de alerta diário e provocando um ciclo vicioso da dor, visto que o stress agrava os sintomas. Segundo dados epidemiológicos, a presença da doença no Brasil é estimada entre 2 e $3 \%$ da população. A prevalência da doença é majoritariamente feminina, ocorrendo em 7,5 mulheres para cada homem (Souza \& Marchand, 2017).

Ela acomete mulheres entre 30 e 55 anos de idade em sua maioria, ocorrendo principalmente em populações que estão em estado de vulnerabilidade social, política e econômica (Mattos \& Cunha, 2017; Souza \& Perissinotti, 2018). Além do sofrimento físico, há um sofrimento psicológico com ocorrência de transtornos depressivos e ansiosos. A dor, mais do que um fenômeno fisiológico, é também uma "experiência subjetiva" (Zaki, Wager, Singer, Keysers \& Gazzola, 2016) e intervenções puramente biológicas nem sempre são eficientes para eliminação ou redução da dor (Epker \& Block, 2001). Assim, para que a FM seja devidamente abordada é importante compreender o indivíduo como um todo, observando sua história com a dor, aspectos emocionais e cognitivos, relação com o ambiente, capacidade funcional, impacto na qualidade de vida e doenças associadas (Coutinho \& Kobayashi, 2017).

O objetivo deste trabalho foi sumarizar os principais achados de estudos em bancos de dados nacionais e construir um panorama descritivo sobre aspectos psicológicos de pacientes com fibromialgia.

\section{Método}

\section{Procedimentos}

Trata-se de uma revisão integrativa (Souza, Silva \& Carvalho, 2010) a partir de levantamento bibliográfico sobre aspectos psicológicos associados a pacientes com FM. A pesquisa foi feita a partir da questão norteadora "Quais os aspectos psicológicos mais comuns relacionados à fibromialgia?". As buscas foram feitas considerando conteúdo dos títulos e resumos, dentro do período de 2015 a 2019, nos bancos de dados Scielo e Pepsic. Foram utilizados os seguintes descritores em português e inglês: Fibromialgia AND ansiedade; Fibromialgia AND sintomatologia AND ansiosa; Fibromialgia AND depressão; Fibromialgia AND sintomatologia AND depressiva; Fibromialgia AND psicologia; Fibromialgia AND stress; Fibromialgia AND psicológicos; Fibromialgia AND suicí- dio; Fibromialgia AND sexualidade; Fibromyalgia AND anxiety; Fibromyalgia AND anxious; Fibromyalgia AND depression; Fibromyalgia AND depressive; Fibromyalgia AND psychology; Fibromyalgia AND stress; Fibromyalgia AND psychological; Fibromyalgia AND suicide; Fibromyalgia AND sexuality.

Utilizou-se o software State of the Art through Systematic Review - START (Fabbri, Silva, Hernandes, Octaviano, Thommazo \& Belgamo, 2016) para a sistematização das etapas de trabalho. Os artigos tiveram seus resumos lidos e foram selecionados aqueles que apresentaram os seguintes critérios de Inclusão: a) Relação da fibromialgia com algum aspecto ou quadro psicológico, b) texto em inglês, português ou espanhol, c) publicado entre os anos de 2015-2019. O levantamento inicial totalizou 96 artigos. Destes, 60 eram duplicados, restando, portanto, 36 artigos, que tiveram seus resumos lidos. 11 foram excluídos por não estabelecerem relações entre fibromialgia e aspectos psicológicos, restando 25 artigos que foram lidos por completo para serem avaliados quanto sua pertinência ao estudo. Por fim, foram selecionados 19 artigos, que tiveram seus dados analisados segundo a recomendação PRISMA (Ver Figura 1).

\section{Análise de Dados}

Os artigos extraídos foram lidos e tabulados (Tabela 1) com informações como Nome do Estudo, Autoria, Ano, Amostra, Instrumentos, Objetivo e Resultados referentes aos aspectos psicológicos da fibromialgia. Depois, foram submetidos à análise bibliométrica (Vouga \& Amatucci, 2015) e análise de conteúdo (Bardin, 2008). Por fim, os dados foram tabulados com uma síntese quantitativa e qualitativa com informações como o ano de publicação, tipo de estudo, tamanho e perfil da amostra, objetivos e principais resultados relacionados à pergunta norteadora deste estudo.

\section{Resultados e discussão}

\section{Análise Bibliométrica}

Quatro artigos foram publicados em 2015, cinco em 2016, quatro em 2017, três em 2018 e três em 2019. Cinco foram publicados em português, cinco em inglês, quatro em espanhol, e cinco publicados tanto em português quanto inglês. Quanto ao país de origem, 15 revistas eram brasileiras, duas espanholas, uma chilena e uma colombiana. Os trabalhos tiveram uma média de 3,3 autores por artigo. 


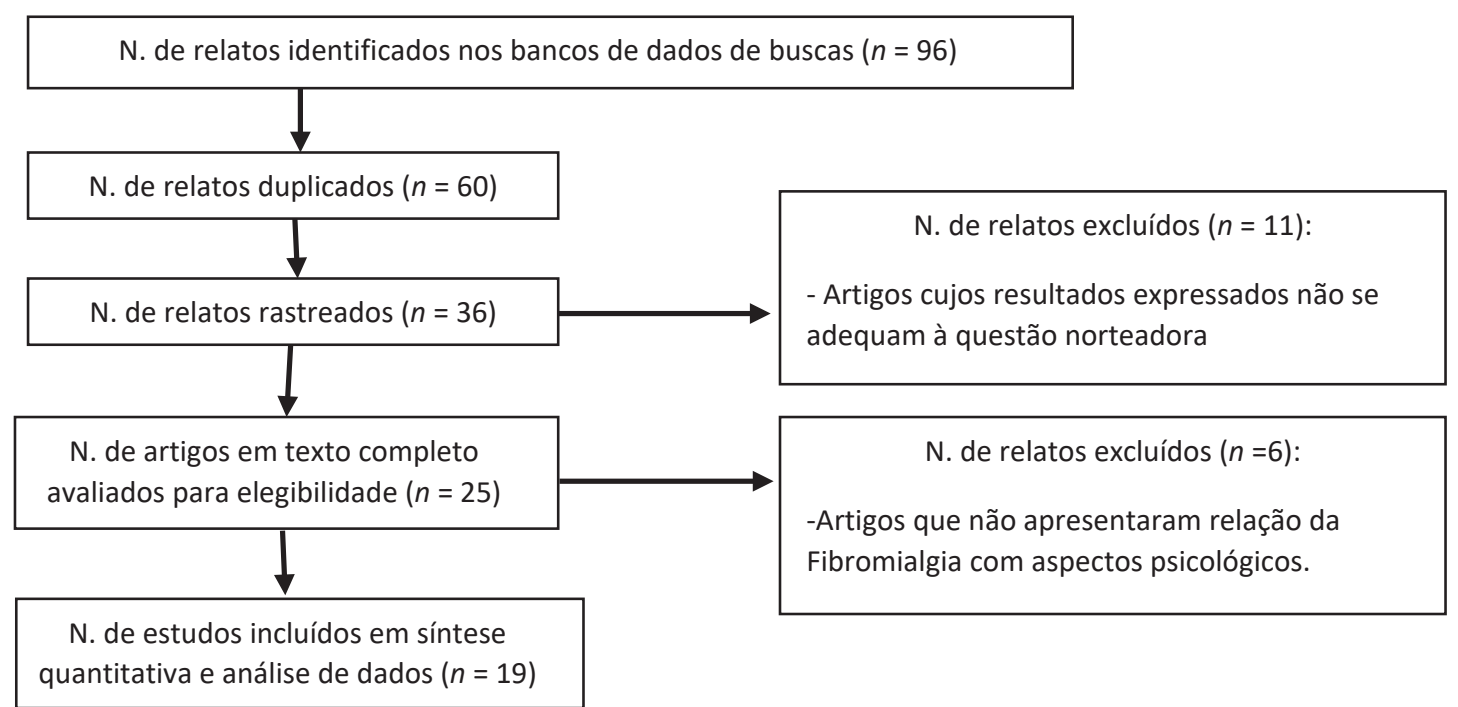

Figura 1: Processo de seleção e análise dos estudos primários encontrados para a revisão integrativa segundo a recomendação PRISMA

Ao separar os artigos em grupos em relação aos métodos de pesquisa, a maioria dos artigos foram de pesquisas de campo $(n=13 ; 68,42 \%)$ e os demais foram de revisão bibliográfica $(n=4 ; 21 \%)$ e estudo psicométrico $(n=2$; $10,52 \%)$. Dentre os estudos de campo, a amostra variou de quatro a 3217, com média de 354,53 e mediana de 98 .

Nos 13 artigos de campo, a população foi totalmente feminina em sete artigos, e predominantemente em seis, confirmando o que se observa na literatura (Souza \& Marchand, 2017; Mattos \& Cunha, 2017). A mesma predominância feminina também ocorreu nos dois estudos psicométricos. O Inventário de Depressão de Beck - BDI I e II - foi o instrumento mais usado para avaliar depressão $(n=4 ; 31 \%)$, seguido da Escala da Escala de Ansiedade e Depressão Hospitalar - HADS ( $n=3 ; 23 \%)$, que além da depressão, também avalia ansiedade, sendo o mais utilizado para esse fim.

\section{Análise de Conteúdo}

\section{Coping e Estresse}

$\mathrm{Na} F \mathrm{~F}$, diversos processos de enfrentamento são exigidos diante de sua sintomatologia constante e incurável na vida do sujeito. O tema apareceu em oito artigos, ilustrando processos adaptativos e desadaptativos, que podem variar em função do gênero, idade e outras características individuais (Moretti, Silva \& Novoa, 2018; Goulart, Pessoa \& Lombardi-Junior, 2015; Dias, Marques, Bettini e Paiva, 2017; Sarudiansky, 2016; Souza \& Laurenti, 2017; Oliveira, Berardinelli, Cavaliere, Rosa, Costa \& Barbosa, 2019; Danet, Prieto-Rodríguez, Valcárcel-
-Cabrera \& March-Cerdá, 2016; Macedo, Martins-Silva, Gavi \& Macedo, 2015).

Foi relatado em três artigos (Moretti, Silva \& Novoa, 2018; Souza \& Laurenti, 2017; Oliveira et al., 2019) o abandono de práticas saudáveis e de autocuidado, devido ao medo e incerteza de piora do quadro de saúde, ocorrendo o isolamento social como estratégia consequente. Partindo de uma análise de gênero, o coping da mulher apresenta adversidades pela maneira como nossa cultura lida com a divisão de tarefas domésticas. Com isso, ela sofre pela falta de estabelecimento e compreensão de limites impostos, sendo potencializados pela doença (Souza \& Laurenti, 2017; Oliveira et al., 2019; Danet, Prieto-Rodríguez, Valcárcel-Cabrera \& March-Cerdá, 2016; Macedo, Martins-Silva, Gavi \& Macedo, 2015). No que tange à realidade de homens, ainda que sejam minoria na doença, é referido um pior manejo psicológico em relação às mulheres (Danet, Prieto-Rodríguez, Valcárcel-Cabrera \& March-Cerdá, 2016).

Com relação à idade, jovens com FM, quando comparados a outros grupos, têm problemas específicos no manejo da doença com reações desadaptativas como absenteísmo escolar e redução de atividade física, por causa da experiência aversiva da dor em seu cotidiano (Goulart, Pessoa \& Lombardi-Junior, 2015).

De maneira geral, o modo como se percebe a doença e sua gravidade interfere na adesão ao tratamento. Aqueles que caracterizam a doença como mais grave tendem a apresentar comportamentos de enfrentamento piores e maior risco de desenvolver transtornos como a depressão (Goulart, Pessoa \& Lombardi-Junior, 2015). 
Tabela 1: Panorama descritivo dos dados coletados

\begin{tabular}{|c|c|c|c|c|c|}
\hline \multirow[b]{2}{*}{$\begin{array}{l}\text { Autoria (ano). Título } \\
\text { do Estudo. }\end{array}$} & \multicolumn{3}{|c|}{ Análise Bibliométrica } & \multicolumn{2}{|c|}{ Análise de Conteúdo } \\
\hline & $\begin{array}{l}\text { Tipo de } \\
\text { Estudo }\end{array}$ & $\begin{array}{l}\text { Amostra } \\
\text { (n e sexo) }\end{array}$ & Instrumentos* & Objetivo & $\begin{array}{l}\text { Principais resultados } \\
\text { referentes aos aspec- } \\
\text { tos psicológicos da } \\
\text { Fibromialgia }\end{array}$ \\
\hline
\end{tabular}

\begin{tabular}{|c|c|c|c|c|c|}
\hline $\begin{array}{l}\text { Moretti, Silva e Novoa } \\
\text { (2018). Characteristics } \\
\text { and perception of so- } \\
\text { cial support by patients } \\
\text { with fibromyalgia in } \\
\text { Facebook. }\end{array}$ & $\begin{array}{l}\text { Pesq. de } \\
\text { Campo }\end{array}$ & $\begin{array}{c}3217 \\
(31369 \\
\left.810^{7}\right)\end{array}$ & ESSS & $\begin{array}{l}\text { Analisar interação, } \\
\text { características, percep- } \\
\text { ção de apoio social e } \\
\text { interesses de pacientes } \\
\text { com fibromialgia em } \\
\text { grupos online. }\end{array}$ & $\begin{array}{l}\text { Relação do suporte } \\
\text { social positivo à me- } \\
\text { lhora de sintomas; falta } \\
\text { de atividades sociais e } \\
\text { de alguém íntimo para } \\
\text { desabafar; laços sociais } \\
\text { fragilizados devido } \\
\text { ao isolamento. Sinto- } \\
\text { mas mais citados: dor, } \\
\text { ansiedade, problema de } \\
\text { memória, irritabilidade e } \\
\text { formigamento. }\end{array}$ \\
\hline $\begin{array}{l}\text { Goulart, Pessoa e } \\
\text { Lombardi-Junior } \\
\text { (2015). Aspectos psi- } \\
\text { cológicos da síndrome } \\
\text { da fibromialgia juvenil: } \\
\text { revisão de literatura. }\end{array}$ & $\begin{array}{l}\text { Rev. biblio- } \\
\text { gráfica }\end{array}$ & & Não se aplica & $\begin{array}{l}\text { Investigar fatores psi- } \\
\text { cossociais relacionados } \\
\text { com a Síndrome da } \\
\text { Fibromialgia Juvenil. }\end{array}$ & $\begin{array}{l}\text { Percepção de menor } \\
\text { gravidade da FM indica } \\
\text { melhor adesão ao trata- } \\
\text { mento; maiores níveis } \\
\text { de fadiga e distúrbios } \\
\text { do sono. Pior qualidade } \\
\text { de vida, comportamen- } \\
\text { tos disfuncionais e mais } \\
\text { sintomas depressão e } \\
\text { ansiedade em relação a } \\
\text { outros grupos. }\end{array}$ \\
\hline $\begin{array}{l}\text { Pernambuco, Sil- } \\
\text { va, Fonseca, Reis e } \\
\text { D’Ávila (2017). Clinical } \\
\text { profile of patients with } \\
\text { fibromyalgia syndrome. }\end{array}$ & $\begin{array}{l}\text { Pesq. de } \\
\text { Campo }\end{array}$ & 76 웅 & $\begin{array}{c}\text { PFS; PSQI; BAI; } \\
\text { BDI }\end{array}$ & $\begin{array}{l}\text { Mensurar e comparar } \\
\text { os níveis de fadiga, } \\
\text { distúrbios do sono, an- } \\
\text { siedade e depressão em } \\
\text { pacientes com FM. }\end{array}$ & $\begin{array}{l}\text { Fadiga, sono e maiores } \\
\text { níveis de ansiedade e } \\
\text { depressão relaciona- } \\
\text { dos à presença da FM; } \\
\text { fadiga correlacionada } \\
\text { à depressão, e ambas } \\
\text { predizendo aumento } \\
\text { de distúrbios do sono, } \\
\text { ansiedade, e sintomas } \\
\text { da FM, como a dor. }\end{array}$ \\
\hline $\begin{array}{l}\text { Freitas, Andrade, Spyri- } \\
\text { des, Micussi e Sousa } \\
\text { (2016). Impacto do } \\
\text { apoio social sobre os } \\
\text { sintomas de mulheres } \\
\text { brasileiras com fibro- } \\
\text { mialgia. }\end{array}$ & $\begin{array}{l}\text { Pesq. de } \\
\text { Campo }\end{array}$ & $66+$ & $\begin{array}{l}\text { MOS-SSS; FIQ; } \\
\text { HAS; Panas; LDP } \\
\text { e TAP }\end{array}$ & $\begin{array}{l}\text { Avaliar o impacto de } \\
\text { suporte social nos } \\
\text { sintomas de mulheres } \\
\text { brasileiras com FM. }\end{array}$ & $\begin{array}{l}\text { Pacientes com menor } \\
\text { apoio social tem piores } \\
\text { índices de depressão, } \\
\text { afeto negativo e sensi- } \\
\text { bilidade a dor; suporte } \\
\text { social contribui com a } \\
\text { melhora mental e física } \\
\text { na FM. }\end{array}$ \\
\hline
\end{tabular}


Análise Bibliométrica

Autoria (ano). Título do Estudo.

\begin{tabular}{|c|c|c|c|}
\hline \multicolumn{3}{|c|}{ Análise Bibliométrica } & Análise d \\
\hline $\begin{array}{l}\text { Tipo de } \\
\text { Estudo }\end{array}$ & $\begin{array}{c}\text { Amostra } \\
\text { (n e sexo) }\end{array}$ & Instrumentos* & Objetivo \\
\hline $\begin{array}{l}\text { Pesq. de } \\
\text { Campo }\end{array}$ & $\begin{array}{c}347 \\
(333 q ; \precsim 14)\end{array}$ & $\begin{array}{l}\text { VNRS; } \\
\text { PHQ-9. }\end{array}$ & $\begin{array}{l}\text { Avaliar o impacto da } \\
\text { intervenção de enfer- } \\
\text { magem no controle } \\
\text { da dor e de sintomas } \\
\text { depressivos nos pacien- } \\
\text { tes de FM. }\end{array}$ \\
\hline
\end{tabular}

Ali, Sanches, Lauretti e Salvetti (2018). Efeitos de uma intervenção de enfermagem no controle de sintomas de pacientes com fibromialgia. Relato de caso.
Avaliar o impacto da intervenção de enferda dor e de sintomas depressivos nos pacien-
Dias, Marques, Bettini e Paiva (2017). Prevalência de fibromialgia em pacientes acompanhados no ambulatório de cirurgia bariátrica do Hospital de Clínicas do Paraná - Curitiba.
Determinar a prevalência de FM em um grupo de pacientes obesos com indicação de cirurgia bariátrica.
Campo
98 pessoas
$(84 \bigcirc ; 140 \hat{)})$
IGD; EDG; EGS;
ESE;
Principais resultados referentes aos aspectos psicológicos da Fibromialgia

Relação entre a diminuição dos sintomas da FM com a diminuição de sintomas depressivos; diminuição dos casos de depressão moderada e muito grave após diminuição de sintomas.

A depressão foi mais comum nas pacientes com FM; houve relação positiva entre o impacto da FM e o impacto no sono.
Alves, Zakka, Teixeira, Kaziyama, Siqueira e Siqueira (2016). Depression, sexuality and fibromyalgia syndrome: clinical findings and correlation to hematological parameters.
Pesq. de Campo 52 ㅇ
FSFI; BDI; VAS; História médica e exames
Investigar sexualidade e depressão em mulheres com FM. Correlacionar os achados com parâmetros hematológicos.
FM associada com altos índices de disfunção sexual e depressão; redução da qualidade de vida; mecanismos patofisiológicos parecem diminuir os hormônios sexuais, e podendo participar da etiologia da FM.

Pessoas com sintomas mais graves de FM e maior índice de depressão estão em risco de SPM e DP; FM comórbida a quatro condições psiquiátricas: depressão, transtorno do pânico, ansiedade e transtorno de estresse pós-traumático.

Transtornos de ansiedade e depressão enquanto comorbidades da FM; FM pode surgir a partir de fatores como estresse ou sobrecarga; FM citada em alguns trabalhos não como uma enfermidade definida, mas como "mal-estar generalizado”.
Sarudiansky (2016).

la fibromialgia en la prensa escrita Argentina: descripciones generales y el rol de la psicología.
Rev. Bibliográfica Não se aplica a FM na Argentina, analisando descrições da FM e tratamentos para refletir como a psicologia é abordada no tratamento.
Avaliar produção sobre 
Análise Bibliométrica

\author{
Autoria (ano). Título \\ do Estudo.
}

\begin{tabular}{lccc}
\hline & Análise Bibliométrica & Análise d \\
\hline $\begin{array}{l}\text { Tipo de } \\
\text { Estudo }\end{array}$ & $\begin{array}{c}\text { Amostra } \\
\text { (n e sexo) }\end{array}$ & Instrumentos* & Objetivo \\
& & \\
Pesq. de & & \\
Campo & 89 & Ent. Semiestrutu- \\
rada & $\begin{array}{l}\text { Operacionalizar a dor } \\
\text { crônica na FM em ter- } \\
\text { mos de contingências e } \\
\text { comportamentos. }\end{array}$
\end{tabular}

Souza e Laurenti

(2017). Uma Interpretação Molar da Dor Crônica na Fibromialgia. Psicol. cienc. prof.

Descrever o cotidiano das mulheres com FM durante a intervenção

Pesq. de

Campo
Entrevista semiestruturada
Oliveira, Berardinelli, cotidiano de mulheres com fibromialgia e o desafio interdisciplinar de empoderamento para o autocuidado. do grupo interdisci-

plinar e analisar seus benefícios à saúde das mulheres após a intervenção.

Principais resultados referentes aos aspectos psicológicos da Fibromialgia

Incontrolabilidade e limitação corporal, com consequências estressantes, gerando evitação de reprovação social, problemas de atenção, realização malfeita de tarefas; fatores presentes nas participantes antes da FM: baixa autoestima, alta responsabilidade e perfeccionismo.

Presença de sono não reparador na FM; ligação entre FM e estresse. A dor crônica gera exclusão social e sofrimento físico e psicológico. As ações do grupo interdisciplinar geraram benefícios físicos, psicológicos e sociais.

A maioria das pacientes referiu impactos na

Horta-Baas e Romero-Figueroa (2019). Self-reported disability in women with fibromyalgia from a tertiary care center.
Pesq. de Campo $115 ㅇ$

WHODAS 2.0 (36-item version); VAS; HADS; Modified TAS-20
Avaliar a relação entre nível de comprometimento funcional e quatro variáveis: intensidade da dor, depressão, ansiedade e alexitimia. funcionalidade, e o nível de perda foi maior em comparação com outras doenças reumatológicas. Mais da metade apresentou transtornos depressivos, ansiosos e/ ou alexitimia.

Correlação positiva entre ansiedade e depressão com a gravidade dos sintomas da FM. Maior presença de transtornos ansiosos, depressivos e ciclotímicos em pacientes com FM do que no grupo controle saudável. 
Análise Bibliométrica

Autoria (ano). Título do Estudo.

$\begin{array}{lccc}\begin{array}{c}\text { Tipo de } \\ \text { Estudo }\end{array} & \begin{array}{c}\text { Amostra } \\ \text { (n e sexo) }\end{array} & \text { Instrumentos* } & \text { Objetivo }\end{array}$

Análise de Conteúdo

Principais resultados referentes aos aspectos psicológicos da Fibromialgia

A prevalência de ansiedade e depressão em pacientes com fibro-
Cabrera, Martín-Aragón, Terol, Núñez e Pastor (2015). La Escala de Ansiedad y Depresión Hospitalaria (HAD) en fibromialgia: Análisis de sensibilidad y especificidad.
Estudo Psicométrico 131 HADS; BDI; STAI-E
Comprovar a aplicabilidade de propriedades psicométricas e sensibilidade/ especificidade da escala HADS na FM.
Danet, Prieto-Rodríguez, Valcárcel-Cabrera e March-Cerdà (2016). Evaluación de una estrategia formativa entre iguales en fibromialgia: Un análisis de género.
Questionário adaptado de "Programa de Paciente Experto de la Universidad de Stanford"
Calcular e avaliar numa perspectiva de gênero, a estratégia formativa de pacientes com FM.

$\begin{array}{lll}\text { Pesq. de } & 559 & \text { grama de Paciente } \\ \text { Campo } & (98 \% \text {. } & \text { Experto de la } \\ & \jmath 2 \%) & \text { Universidad de } \\ & & \text { Stanford" }\end{array}$
mialgia foi superior a de outros grupos. A HADS apresentou propriedades psicométricas ótimas na população de FM, sendo útil como instrumento de rastreio da sintomatologia ansioso-depressiva na população.

Intervenção melhorou stress, hábitos de vida e manejo da dor e busca por informações, reduzindo visitas médicas.

Homens tem pior manejo da FM. Maior estresse em pessoas com menos de três anos com a FM, principalmente nas que trabalham ou procuram emprego.
Investigar o impacto biológico de técnicas baseadas nos modelos de apego e seu papel nos processos de resiliência.
Nieto (2015). Apego, Cortisol y estrés en adultos, una revisión narrativa. Rev. Asoc. Esp. Neuropsiq.
Revisão Bi-

bliográfica
Não se aplica
Ordoñez-Carrasco,

Salgueiro, Sayans-Ji-

ménez, Blanc-Molina,

García-Leiva, Calandre

e Rojas (2018). Psycho-

metric properties of the Spanish version of the 12-item Interpersonal Needs Questionnaire in fibromyalgia syndrome patients.
Analisar as propriedades psicométricas da adaptação em espanhol do INQ-12 em pacientes com fibromialgia.
Apego inseguro tem relação psicopatológica com o estresse crônico; existe relação entre estresse constante e FM.

Relação direta entre ideação suicida e FM, e construtos como desesperança, sensação de sobrecarga e não pertencimento; relação entre severidade e duração da dor com maiores ideações suicidas; FM ligada a fatores de risco de suicídio.

Existência de um campo de fragilidade das mulheres com fibromialgia e pessoas próximas a elas no enfrentamento da doença; presença de privação de sono na FM; depressão e ansiedade presentes na fala de uma participante. 
Autoria (ano). Título do Estudo.
Análise Bibliométrica

Tipo de

Estudo
Amostra

(n e sexo)
Instrumentos*

Análise de Conteúdo

Principais resultados referentes aos aspectos psicológicos da Fibromialgia

Maiores níveis de distress, depressão e maior quantidade de tender

Centurion e Peres (2016). A sexualidade em pacientes com fibromialgia: panorama da produção científica.
Revisão Bibliográfica Não se aplica
Investigar a sexualidade em pacientes com FM points são preditores

de problemas sexuais; mulheres com FM tem sua qualidade de vida e sexualidade comprometidas.

*Nota: Instrumentos em ordem alfabética. BAI-Beck Anxiety Inventory; BDI-Beck Depression Inventory; EGS- Escala de Gravidade de Sintomas; EGD-Índice de Dor Generalizada; ESE-Escala de Sonolência Epworth; ESSS: Escala de Satisfação com o suporte social; FCSS: Fibromyalgia Criteria and Severity Scales; FIQ: Questionário de Impacto da Fibromialgia; FSFI: Índice de Função Sexual Feminina; HAM-D: Escala de Depressão de Hamilton; HAS: Escala de Ansiedade de Hamilton; HADS: Hospital Anxiety and Depression Scale; LDP: Limiar da dor à pressão; MOS-SSS: Social Suport Survey; Panas: Escala de Afetos positivos e negativos; PFS: Piper Fatigue Scale; PSQI: Pittsburgh Sleep Quality Index; PHQ-9:Patient Health Questionnaire; PSRS: Plutchik Suicide Risk Scale; STAI-E: State Trait Anxiety Inventory; TAP: Tolerância álgica à pressão; TAS-20: Toronto Alexithymia Scale; TEMPS-A: Temperament Scale of Memphis, Pisa and San Diego; VAS: Visual Analogue Scale; VNRS-Escala numérica verbal de dor.

Assim, é importante o foco em intervenções baseadas em evidências que auxiliem nessa adaptação, desde estratégias educativas, acolhimento e convivência entre semelhantes, combinadas à prática de exercícios físicos (Freitas, Andrade, Spyrides, Micussi \& Sousa, 2016; Oliveira et al., 2019; Danet, Prieto-Rodríguez, Valcárcel-Cabrera \& March-Cerdá, 2016; Macedo, Martins-Silva, Gavi \& Macedo, 2015) e medicamentos (Sarudiansky, 2016), visto que a FM costuma apresentar diversas comorbidades psicológicas.

É importante destacar que o fato de lidar com um tratamento multidisciplinar e muitas variáveis, desde a dor até consequências socioeconômicas, limitações físicas e perda de autonomia, deixa os pacientes mais vulneráveis ao estresse (Sarudiansky, 2016; Souza \& Laurenti, 2017; Oliveira et al., 2019; Horta-Baas \& Romero-Figueroa, 2019; Centurion \& Peres, 2016). Alguns artigos citaram-no também como possível fator de agravo da FM, devido a associação com hormônios que em excesso possuem efeitos inflamatórios nocivos (Sarudiansky, 2016; Souza \& Laurenti, 2017; Nieto, 2015).

Assim, a saúde mental é descrita como um elemento que demanda atenção, visto que a FM causa um grande desgaste emocional, com consequentes transtornos psicológicos que por sua vez podem agravar os sintomas ou mesmo alterar a percepção da dor (Horta-Baas \& Romero-Figueroa, 2019). Um dos estudos (Danet, Prieto-
-Rodríguez, Valcárcel-Cabrera \& March-Cerdá, 2016) destaca uma relação de maior estresse em pessoas com menos de três anos de convivência com a enfermidade, e principalmente em mulheres que trabalham fora de casa ou que estão à procura de emprego.

O estresse deve, portanto, ser considerado pelo olhar do psicólogo, pois quando elevado pode piorar os sintomas médicos e levar a quadros depressivos e ansiosos. Diante dessas circunstâncias, é necessário auxiliar o desenvolvimento de estratégias de enfrentamento adequadas às necessidades e contexto de cada pessoa. Com relação ao gênero, além das questões individuais e ligadas à enfermidade e ao tratamento, as mulheres estão expostas a rotinas mais estressantes e acúmulo de tarefas domésticas, por conta de questões culturais e sociais que merecem ser refletidas (Instituto Brasileiro de Geografia e Estatística - [IBGE], 2018; Kuehner, 2017) e que provavelmente explicam a maior frequência de estresse, ansiedade, depressão e outros quadros psicológicos, conforme apresentado adiante.

\section{Depressão}

A depressão foi o transtorno mental com a maior frequência nos artigos, sendo citada em 13 artigos (Goulart, Pessoa \& Lombardi-Junior, 2015; Pernambuco, Silva, Fonseca, Reis \& D’Ávila, 2017; Freitas, Andrade, Spyrides, Micussi \& Sousa, 2016; Ali, Sanches, Lauretti 
\& Salvetti, 2018; Dias, Marques, Bettini e Paiva, 2017; Alves, Zakka, Teixeira, Kaziyama, Siqueira \& Siqueira, 2016; Terzi, Terzi \& Kale, 2015; Sarudiansky, 2016; Horta-Baas \& Romero-Figueroa, 2019; Işık-Ulusoy, 2019; Cabrera, Martín-Aragón, Terol, Núñez \& Pastor, 2015; Ordoñez-Carrasco, Salgueiro, Sayans-Jiménez, Blanc-Molina, García-Leiva, Calandre \& Rojas, 2018; Macedo, Martins-Silva, Gavi \& Macedo, 2015). Os estudos demonstraram relação entre a depressão e a FM quando comparados a grupos controle sem doenças crônicas, pontuando escores mais altos em diversos instrumentos psicológicos. (Dias, Marques, Bettini e Paiva, 2017; Terzi, Terzi \& Kale, 2015; Işı-Ulusoy, 2019; Danet, Prieto-Rodríguez, Valcárcel-Cabrera \& March-Cerdà, 2016; Nieto, 2015). O nível de depressão apresentou correlação com a gravidade dos sintomas da dor (Terzi, Terzi \& Kale, 2015; Iş1k-Ulusoy, 2019) e pode piorar a percepção de sintomas da doença, reduzindo a qualidade de vida do sujeito (Pernambuco, Silva, Fonseca, Reis \& D'Ávila, 2017; Alves et al., 2016; Horta-Baas \& Romero-Figueroa, 2019; Cabrera, Martín-Aragón, Terol, Núñez \& Pastor, 2015), podendo gerar prejuízos na vida sexual (Freitas, Andrade, Spyrides, Micussi \& Sousa, 2016). Isso evidencia a importância de o psicólogo e outros profissionais de saúde avaliarem a adesão ao tratamento, e da realização de trabalhos multidisciplinares, visto que somente uma forma de tratamento não é suficiente para dar conta da complexidade da enfermidade, conforme destacam Perissinotti e Sardá-Junior (2019).

Embora a relação entre a depressão e a FM não esteja perfeitamente compreendida enquanto mecanismo neurofisiológico, como explicitado por Terzi, Terzi e Kale (2015), acredita-se que a dor crônica possa causar a depressão. Uma hipótese para essa relação pode ser o desamparo aprendido (Seligman, 1975), em que o indivíduo perde a sensação de controle de estímulos aversivos, tendendo a apresentar transtornos psicológicos, em especial, a depressão devido a exposição frequente a uma situação estressora. Moyano, Scolnik, Vergara, García, Sabelli, Rosa, Catoggio e Soriano (2018) destacam que este desamparo é maior em pacientes com doenças cujos sintomas geram um impacto maior na qualidade de vida ativa, como ocorre em pacientes com FM, em que se observam dor, fadiga, depressão e menor autoeficácia do que naqueles com outras enfermidades.

Além disso, especula-se que as síndromes de dor crônica possam ser variantes de sintomas da depressão em si - visto que o transtorno afeta a psicomotricidade do corpo -, ocorrendo nesses dois processos separadamente ou concomitantemente, mas sem poder afirmar causa e efeito entre eles (Dias, Marques, Bettini e Paiva, 2017; Terzi, Terzi e Kale, 2015).

O uso de antidepressivos faz parte do tratamento da FM e em pesquisa de Ali, Sanches, Lauretti e Salvetti (2018), pouco mais da metade da amostra pesquisada utilizava medicamentos. Levando em conta a importância desse tratamento para a qualidade de vida do paciente, é imprescindível uma maior adesão. Tal fato remete às reações desadaptativas em relação ao tratamento, conforme destacado anteriormente (Ali, Sanches, Lauretti \& Salvetti, 2018; Sarudiansky, 2016).

Foi observada ainda uma relação entre maior frequência de ideações suicidas e a FM, havendo o risco de suicídio pela sensação de sobrecarga, não pertencimento e desesperança nos pacientes, devido aos sintomas da doença. Percebeu-se relação direta de ideação suicida com severidade e duração da dor (Ordoñez-Carrasco et al., 2018). Variáveis sociais também foram citadas, como o apoio social negativo, que teve correlação positiva quando relacionado à depressão, baixando os níveis da mesma e melhorando os sintomas conforme a pessoa tem mais interações sociais (Freitas, Andrade, Spyrides, Micussi \& Sousa, 2016), sendo paradoxal com o fato de que a depressão é preditora de dificuldades nas relações sociais (Horta-Baas \& Romero-Figueroa, 2019).

Em dois artigos (Sarudiansky, 2016; Işı-Ulusoy, 2019), a ansiedade e a depressão foram citadas como comorbidades próprias da sintomatologia da FM. Outras condições pouco citadas, mas que apresentaram relação significativa com a depressão e a FM foram: Alexitimia (Horta-Baas \& Romero-Figueroa, 2019), Ciclotimia (Iş1kUlusoy, 2019) e Síndrome Pré-Menstrual e Dismenorreia Primaria (Terzi, Terzi \& Kale, 2015).

\section{Ansiedade}

A ansiedade foi o segundo tema mais citado na revisão, sendo citada em oito artigos (Moretti, Silva \& Novoa, 2018; Goulart, Pessoa \& Lombardi-Junior, 2015; Dias, Marques, Bettini e Paiva, 2017; Terzi, Terzi \& Kale, 2015; Sarudiansky, 2016; Işık-Ulusoy, 2019; Cabrera, Martín-Aragón, Terol, Núñez \& Pastor, 2015; Macedo, Martins-Silva, Gavi \& Macedo, 2015). Ela é ligeiramente menos presente do que a depressão nos pacientes (Danet, Prieto-Rodríguez, Valcárcel-Cabrera \& March-Cerdà, 2016), mas é importante destacar, conforme observado em três estudos (Goulart, Pessoa \& Lombardi-Junior, 2015; Terzi, Terzi \& Kale, 2015; Sarudiansky, 2016), que os pacientes com FM são mais ansiosos que a população 
em geral, e que essa ansiedade está relacionada também ao quão mais severos esses sintomas são (Ișik-Ulusoy, 2019). Galvez-Sánchez, Montoro, Duschek e Reyes Del Paso (2020) confirmam a presença da ansiedade e depressão na FM, com relação direta à gravidade dos sintomas e destacam os riscos de o paciente entrar em um ciclo vicioso, no qual os sintomas geram mais transtornos psicológicos, que por sua vez podem interferir na sintomatologia da FM.

A ansiedade é um estado emocional que nos prepara para uma ameaça. Porém, em indivíduos considerados ansiosos, geralmente ela está associada a uma perspectiva errônea com uma probabilidade superestimada de ocorrência de um dano ou agravo. Não conseguem perceber os aspectos que poderiam gerar segurança e subestimam sua capacidade de enfrentar dano ou perigo antecipado (Clark \& Beck, 2012). No caso da FM, é comum a antecipação de uma possível experiência estressante ou dolorosa, o que reforça a importância do controle álgico, da adesão ao tratamento e do acompanhamento psicológico para auxiliá-lo a ter expectativas mais realistas e funcionais.

\section{Outros aspectos psicológicos}

Existem outros aspectos psicológicos que foram menos frequentes nos estudos levantados, mas que merecem destaque pelo prejuízo que podem causar à qualidade de vida do paciente. O impacto da FM no sono foi relatado em quatro artigos (Goulart, Pessoa \& Lombardi-Junior, 2015; Dias, Marques, Bettini e Paiva, 2017; Oliveira et al., 2019; Macedo, Martins-Silva, Gavi \& Macedo, 2015), e suas possíveis consequências, como a fadiga, em três (Goulart, Pessoa \& Lombardi-Junior, 2015; Pernambuco, Silva, Fonseca, Reis \& D’Ávila, 2017; Danet, Prieto-Rodríguez, Valcárcel-Cabrera \& March-Cerdà, 2016). As alterações cognitivas foram citadas em três (Moretti, Silva \& Novoa, 2018; Dias, Marques, Bettini e Paiva, 2017; Souza \& Laurenti, 2017). Esses três fatores estão interligados em pacientes de dor crônica, visto que a falta do sono reparador gera diversos problemas cognitivo-emocionais (prejudicando memória, concentração e aumentando a irritabilidade) e psicofísicos (como fadiga e sobrecarga) pela falta do descanso (Souza \& Marchand, 2017).

Três estudos levantados (Dias, Marques, Bettini e Paiva, 2017; Oliveira et al., 2019; Macedo, Martins-Silva, Gavi \& Macedo, 2015) apontam que a privação do sono ocorre de forma proporcional ao nível de impacto da doença, afetando a qualidade dele, podendo ser fragmentando e leve, ou ainda caracterizar-se como insônia em casos mais graves. Foi correlacionado estatisticamente em um dos estudos (Pernambuco, Silva, Fonseca, Reis \& D’Ávila, 2017) que a fadiga e a depressão também são capazes de alterar outros sintomas clínicos da doença, como a ansiedade e os distúrbios do sono anteriormente explicitados.

A FM também interfere na vida sexual dos pacientes (Alves et al., 2016; Macedo, Martins-Silva, Gavi \& Macedo, 2015; Centurion \& Peres, 2016), que podem ter experiências dolorosas antes, durante e após o ato, com profundas consequências emocionais. Ocorre também a diminuição da libido, por conta de medicações e mudanças hormonais (Alves et al., 2016; Macedo, Martins-Silva, Gavi \& Macedo, 2015) e do próprio sofrimento gerado pelo ato. Essa mudança na dinâmica leva a conflitos na vida conjugal, devido ao não entendimento do parceiro sobre a doença do cônjuge - seja por falta de informação ou falta de aceitação da mesma - e à autocobrança da mulher (Macedo, Martins-Silva, Gavi \& Macedo, 2015).

Mulheres com FM têm maior inatividade sexual do que aquelas com outras doenças crônicas, como lúpus (Centurion \& Peres, 2016). Foi apontado pelo mesmo artigo que mais distress atua como preditor de disfunções sexuais, porém é um fator pouco estudado em homens devido à menor proporção da doença nessa população.

\section{Considerações finais}

O objetivo desse artigo foi sumarizar os principais achados de estudos em bancos de dados nacionais para construir um panorama descritivo sobre aspectos psicológicos de pacientes com FM. Para isso, foi utilizado o método da revisão integrativa, para poder acessar os dados de forma mais rápida e poder aplicar na vivência clínica, porém, sem perder as análises quantitativas e qualitativas.

A análise dos dados apontou a presença de sofrimento psicológico na vivência de pacientes com FM, com destaque para os quadros ansiosos e depressivos. Esse sofrimento chama atenção pelo potencial que o estresse tem de gerar repostas inflamatórias, desencadeando uma reação em cadeia no organismo. Na condição de fenômeno multidimensional, a FM demanda um processo complexo de tratamento e compreensão do paciente, envolvendo controle de sintomas, implementação de novos hábitos (como exercícios físicos), tratamento medicamentoso, entre outros. Isso muitas vezes pode ser afetado por reações desadaptativas, pensamentos disfuncionais, fuga e isolamento social, que podem ampliar ainda mais o sofrimento. Cumpre destacar ainda uma preocupação 
com a população feminina acometida pela FM, além de serem a maioria afetada, sua vivência da enfermidade é atravessada por problemas sociais, culturais e de gênero, que muitas vezes dificultam o estabelecimento e manutenção de limites protetivos à dor, como se observa na precária divisão de tarefas de muitos lares brasileiros ou mesmo na realidade de mães solos.

Essas questões evidenciam a importância de um olhar integral e um acompanhamento multidisciplinar, incluindo a Psicologia, para que o paciente possa lidar com os novos desafios, adaptando-se da forma mais saudável possível à nova rotina, além de tratar de eventuais transtornos psiquiátricos, tão comuns a essa enfermidade. A Psicologia pode colaborar com uma melhor adesão ao tratamento, além de um melhor manejo de dificuldades e comorbidades impostas pela doença, através de ações cientificamente comprovadas, reduzindo o impacto da enfermidade e promovendo saúde mental e mais qualidade de vida.

Espera-se que esse trabalho contribua na prática profissional de psicólogos e profissionais de saúde que lidem com essa realidade. Uma dificuldade foi o foco predominante biomédico na literatura sobre a FM, sendo escassa quando envolve a Psicologia. Dessa forma, optou-se por uma busca mais abrangente sobre aspectos psicológicos. Para estudos posteriores, sugere-se uma revisão sistemática em bancos de dados internacionais, investigando a viabilidade de focar em aspectos ou transtornos psicológicos específicos. Faz-se importante ainda a ampliação de pesquisas de campo que abordem melhor a interação de fenômenos psicológicos com a dor crônica, em especial à FM. De igual modo, o psicólogo deve atentar a essa interação em sua prática clínica e institucional com esses pacientes, priorizando sempre que possível uma abordagem interdisciplinar.

\section{Referências}

Ali, Y. C. M. M., Sanches, M. B., Lauretti, L. G. \& Salvetti, M. de G. (2018). Efeitos de uma intervenção de enfermagem no controle de sintomas de pacientes com fibromialgia. Relato de caso. Brazilian Journal of Pain, 1(4), 365-368. https://doi.org/10.5935/2595-0118.20180069

Alves, B., Zakka, T. M., Teixeira, M. J., Kaziyama, H. H., Siqueira, J. T. T. \& Siqueira, S. R. D. T. (2016). Depressão e sexualidade na síndrome fibromiálgica: Achados clínicos e correlação com parâmetros hematológicos. $A r$ quivos de Neuro-Psiquiatria, 74(11), 863-868. https://doi.org/10.1590/0004$-282 \mathrm{X} 20160141$

Bardin, L. (2008). Análise de conteúdo. Coimbra: Edições 70.

Cabrera, V., Martín-Aragón, M., Terol, M. del C, Núñez, R. \& Pastor, M. A. (2015). Hospital Anxiety and Depression Scale (HADS) in fibromyalgia: sensitivity and specificity analysis. Terapia psicológica, 33(3), 181-193. https:// dx.doi.org/10.4067/S0718-48082015000300003

Centurion, B. N. \& Peres, R. S. (2016). A sexualidade em pacientes com fibromialgia: panorama da produção científica. Rev. SPAGESP, 17(2), 108-119. Recuperado em 07 de julho de 2020, de http://pepsic.bvsalud.org/scielo. php?script $=$ sci_arttext\&pid $=$ S1677-29702016000200009\&lng $=$ pt\&tlng $=$ pt. Clark, D. A. \& Beck, A. T. (2012). Terapia cognitiva para os transtornos de ansiedade. Porto Alegre: Artmed.

Coutinho, M. R. \& Kobayashi, C. (2017). Avaliação psicológica. In Posso, I. P. (Org.). Tratado de dor (pp. 469-476). São Paulo: Atheneu.

Danet, A., Prieto-Rodríguez, M. Á., Valcárcel-Cabrera, M. C. \& March-Cerdà, J. C. (2016). Avaliação de uma estratégia formadora entre pessoas com fibromialgia. Uma análise de gênero. Aquichan, 16(3), 296-312. https:// doi.org/10.5294/aqui.2016.16.3.3

Dias, D. N. G., Marques, M. A. A., Bettini, S. C., \& Paiva, E. dos S. (2017). Prevalência de fibromialgia em pacientes acompanhados no ambulatório de cirurgia bariátrica do Hospital de Clínicas do Paraná $\square$ Curitiba. Revista Brasileira de Reumatologia, 57(5), 425-430. https://doi.org/10.1016/j. rbr.2017.01.001

Epker, J. \& Block, A. R. (2001). Presurgical psychological screening in back pain patients: a review. Clinical Journal of Pain, 17(3), 200-205. https://doi. org/10.1097/00002508-200109000-00003

Fabbri, S., Silva, C., Hernandes, E., Octaviano, F., Di Thommazo, A. \& Belgamo, A. (2016). Improvements in the StArt tool to better support the systematic review process. Proceedings of the 20th International Conference on Evaluation and Assessment in Software Engineering (EASE 16), 21,1-5. https:// doi.org/10.1145/2915970.2916013

Freitas, R. P. A., Andrade, S. C., Spyrides, M. H. C., Micussi, M. T. A. B. C. \& Sousa, M. B. C. (2016). Impacto do apoio social sobre os sintomas de mulheres brasileiras com fibromialgia. Revista Brasileira de Reumatologia, 57(3), 197-203. https://doi.org/10.1016/j.rbr.2016.05.002

Galvez-Sánchez, C. M., Montoro, C. I., Duschek, S. \& Reyes del Paso, G. A. (2020). Depression and trait-anxiety mediate the influence of clinical pain on health-related quality of life in fibromyalgia. Journal of Affective Disorders, 265, 486-495. https://doi.org/10.1016/j.jad.2020.01.129

Goulart, R., Pessoa, C. \& Lombardi-Junior, I. (2015). Aspectos psicológicos da síndrome da fibromialgia juvenil: revisão de literatura. Revista Brasileira de Reumatologia, 56(1), 69-74. https://doi.org/10.1016/j.rbr.2015.07.008

Horta-Baas, G. \& Romero-Figueroa, M. D. S. (2019). Self-reported disability in women with fibromyalgia from a tertiary care center. Advances in Rheumatology, 59(1), 1-9. https://doi.org/10.1186/s42358-019-0086-4

Instituto Brasileiro de Geografia e Estatística [IBGE]. (2018). Estatísticas de gênero: indicadores sociais das mulheres no Brasil. Recuperado em $10 \mathrm{de}$ setembro de 2020 de https://biblioteca.ibge.gov.br/visualizacao/livros/ liv101551_informativo.pdf

Işı1k-Ulusoy, S. (2019). Evaluation of affective temperament and anxiety-depression levels in fibromyalgia patients: A pilot study. Brazilian Journal of Psychiatry, 41(5), 428-432. https://doi.org/10.1590/1516-4446-2018-0057

Kuehner, C. (2017). Why is depression more common among women than among men? The Lancet Psychiatry, 4(2), 146-158. https://doi.org/10.1016/ S2215-0366(16)30263-2

Macedo, D. C. F., Martins-Silva, P. O., Gavi, M. B. R. \& Macedo, A. P. D. (2015). Representações sociais de conjugalidade e fibromialgia: desdobramentos na dinâmica conjugal. Temas em Psicologia, 23(4), 987-1002. https:// doi.org/10.9788/TP2015.4-14

Mattos, P. F. \& Cunha, P. M. (2017). Avaliação Psiquiátrica do paciente com dor crônica. In Posso, I. de P. (Org.), Tratado de Dor (pp. 477-483). São Paulo: Atheneu.

Moretti, F. A., Silva, S. S. \& Novoa, C. G. (2018). Characteristics and perception of social support by patients with fibromyalgia in Facebook. Brazilian Journal of Pain, 1(1), 4-8. https://doi.org/10.5935/2595-0118.20180003

Moyano, S., Scolnik, M., Vergara, F., García, M. V., Sabelli, M. R., Rosa, J. E., Catoggio, L. J. \& Soriano, E. R. (2018). Evaluation of learned helplessness, perceived self-efficacy, and functional capacity in patients with fibromyalgia and rheumatoid arthritis. Journal of Clinical Rheumatology, 25(2), 65-68. https://doi.org/10.1097/RHU.0000000000000769

Nieto, E. A. R. (2015). Apego, cortisol y estrés en adultos, una revisión narrativa. Revista de La Asociación Española de Neuropsiquiatría, 35(125), 53-77. https://doi.org/10.4321/s0211-57352015000100005

Oliveira, J. P. R., Berardinelli, L. M. M., Cavaliere, M. L., Rosa, R. C. A., Costa, L. P. \& Barbosa, J. S. de O. (2019). O cotidiano de mulheres com fibromialgia e o desafio interdisciplinar de empoderamento para o autocuidado. Revista Gaúcha de Enfermagem, 40, e20180411. https://doi.org/10.1590/19831447.2019.20180411 
Ordoñez-Carrasco, J. L., Salgueiro, M., Sayans-Jiménez, P., Blanc-Molina, A., García-Leiva, J. M., Calandre, E. P. \& Rojas, A. J. (2018). Psychometric properties of the Spanish version of the 12-item Interpersonal Needs Questionnaire in fibromyalgia syndrome patients. Anales de Psicología, 34(2), 274. https://doi.org/10.6018/analesps.34.2.293101

Perissinotti, D. M. N. \& Sardá-Junior, J. (2019). Tratamentos psicológicos e dor/ diferentes técnicas e estratégias clínicas e suas aplicações: tratar o quê, para quê e como? In Perissinotti, D. M. N. \& Sardá-Junior, J. (Orgs.), Psicologia e Dor - o que você deve saber (pp. 105-125). São Paulo: Editora dos Editores.

Pernambuco, A. P., Silva, L. R. T., Fonseca, A. C. S. \& Reis, D. d’Ávila. (2017). Clinical profile of patients with fibromyalgia syndrome. Fisioterapia em Movimento, 30(2), 287-296. http://dx.doi.org/10.1590/1980-5918.030.002.ao09

Sarudiansky, M. (2016). A análise temática da fibromialgia na mídia impressa argentina: Descrições gerais e o papel da psicologia. Interface: Communication, Health, Education, 20(56), 25-36. https://doi.org/10.1590/180757622015.0008

Seligman, M. E. P. (1975). Helplessness on depression, development, and death. Londres: W. H. Freeman and Company.

Souza, J. B. \& Marchand, S. (2017). Fibromialgia. In Posso, I. de P., Grossman, E., Fonseca, P. R. B. da, Perissinotti, D. M. N., Oliveira- Júnior, J. O., Souza, J. B. de, Serrano, S.C. \& Vall, J. (Eds.). Tratado de dor (pp. 561-576). São Paulo: Atheneu.

Souza, B. \& Laurenti, C. (2017). Uma interpretação molar da dor crônica na fibromialgia. Psicologia: Ciência e Profissão, 37(2), 363-377. https://doi. org/10.1590/1982-3703001102016
Souza, J. B. \& Perissinotti, D. M. N. (2018). The prevalence of fibromyalgia in Brazil - a population-based study with secondary data of the study on chronic pain prevalence in Brazil. Brazilian Journal of Pain, 1(4), 345-348. https://doi.org/10.5935/2595-0118.20180065

Souza, M. T., Silva, M. D. \& Carvalho, R. (2010). Revisão integrativa: o que é e como fazer. Einstein, 8(1), 102-106.https://doi.org/10.1590/s1679-45082010rw1134

Terzi, R., Terzi, H. \& Kale, A. (2015). Avaliação da relação entre síndrome pré-menstrual e dismenorreia primária em mulheres com fibromialgia. Revista Brasileira de Reumatologia, 55(4), 334-339. https://doi.org/10.1016/j. rbr.2014.12.009

Vouga, G. \& Amatucci, M. (2015). O que é bibliometria? Uma introdução ao Fórum. Revista Eletrônica de Negócios Internacionais, 10, 1-5. https://doi. org/10.18568/1980-48651021-52015

Zaki, J., Wager, T. D., Singer, T., Keysers, C. \& Gazzola, V. (2016). The anatomy of suffering: understanding the relationship between nociceptive and empathic pain. Trends in Cognitive Sciences, 20(4), 249-259. https://doi. org/10.1016/j.tics.2016.02.003

Submetido em: 16-9-2020

Aceito em: 19-4-2021 